\title{
Micro-computed tomography, scanning electron microscopy and energy X-ray spectroscopy studies of facet joint degeneration : A comparison to clinical imaging
}

\author{
Göhre, Felix
}

2017-09

Göhre , F , Ludtka , C , Hamperl , M , Friedmann , A, Straube , A, Mendel , T , Heilmann , A , Meisel , H J \& Schwan, S 2017 , ' Micro-computed tomography, scanning electron microscopy and energy X-ray spectroscopy studies of facet joint degeneration : A comparison to clinical imaging ' , Micron , vol. 100 , pp. 50-59 . https://doi.org/10.1016/j.micron.2017.04.011

http://hdl.handle.net/10138/237201

https://doi.org/10.1016/j.micron.2017.04.011

publishedVersion

Downloaded from Helda, University of Helsinki institutional repository.

This is an electronic reprint of the original article.

This reprint may differ from the original in pagination and typographic detail.

Please cite the original version. 


\title{
Micro-computed tomography, scanning electron microscopy and energy X- ray spectroscopy studies of facet joint degeneration: A comparison to clinical imaging
}

\author{
Felix Goehre ${ }^{\mathrm{a}, \mathrm{b}}$, Christopher Ludtka ${ }^{\mathrm{c}}$, Melanie Hamperl ${ }^{\mathrm{d}}$, Andrea Friedmann ${ }^{\mathrm{e}}$, Anja Straube ${ }^{\mathrm{f}}$, \\ Thomas Mendel $^{g}$, Andreas Heilmann ${ }^{\mathrm{e}}$, Hans Jörg Meisel ${ }^{\mathrm{a}}$, Stefan Schwan ${ }^{\mathrm{e}, *}$ \\ a BG Klinikum Bergmannstrost, Department of Neurosurgery, Halle, Germany \\ b University of Helsinki and Helsinki University Hospital, Department of Neurosurgery, Finland \\ ${ }^{c}$ University of Tennessee, Department of Chemical \& Biomolecular Engineering, Knoxville, TN, USA \\ d Klinikum Stuttgart - Katharinenhospital, Department of Neurosurgery, Stuttgart, Germany \\ e Fraunhofer Institute for Microstructure of Materials and Systems IMWS, Department of Biological and Macromolecular Materials, Halle, Germany \\ ${ }^{\mathrm{f}}$ Ilmenau University of Technology, Group of Biomechatronics, Ilmenau, Germany \\ ${ }^{g}$ Friedrich-Schiller University Jena, Department of Trauma Surgery, Jena, Germany
}

\section{A R T I C L E I N F O}

\section{Keywords:}

Computed tomography

Facet joint

Human lumbar spine

Scanning electron microscopy

Structure analysis

\begin{abstract}
A B S T R A C T
Segmental degeneration in the human lumbar spine affects both the intervertebral discs and facet joints. Facet joint degeneration not only affects the cartilage surface, but also alters the cellular properties of the cartilage tissue and the structure of the subchondral bone. The primary focus of this study is the investigation of these microstructural changes that are caused by facet joint degeneration. Microstructural analyses of degenerated facet joint samples, obtained from patients following operative lumbar interbody fusion, have not previously been extensively investigated. This study analyzes human facet joint samples from the inferior articular process using scanning electron microscopy, micro-computed tomography, and energy dispersive X-ray spectroscopy to evaluate parameters of interest in facet joint degeneration such as elemental composition, cartilage layer thickness and cell density, calcification zone thickness, subchondral bone portion, and trabecular bone porosity. These microstructural analyses demonstrate fragmentation, cracking, and destruction of the cartilage layer, a thickened calcification zone, localized calcification areas, and cell cluster formation as pathological manifestations of facet joint degeneration. The detailed description of these microstructural changes is critical for a comprehensive understanding of the pathology of facet joint degeneration, as well as the subsequent development and efficacy analysis of regenerative treatment strategies.
\end{abstract}

\section{Introduction}

Lower back pain is a major contributing factor to a diminished quality of life, reduced mobility, and financial strain on healthcare systems worldwide (Hoy et al., 2014). One substantial source of lower back pain is attributable to degeneration of the lumbar facet joints (Kalichman and Hunter, 2007; Gellhorn et al., 2013). Posterior lumbar interbody fusion (PLIF) is currently one of the most common methods for alleviating chronic pain from facet joint degradation and involves excision of the affected joints and fixation of the vertebrae with screws and segmental fusion (Lin et al., 1983; Prolo et al., 1986). However, this procedure has the risk of intraoperative and postoperative complications in up to $16 \%$ of cases (Okuyama et al., 1999; Miyakoshi et al.,
2000; DiPaola and Molinari, 2008). Additionally, the surgery both permanently limits patient flexibility and requires significant recovery times (Gill and Blumenthal, 1993). It is therefore pertinent in the scope of medical ethics to verify the necessity of PLIF in cases of lower back pain and facet joint degeneration on an individual basis. Several clinical grading systems have previously been suggested and rely on magnetic resonance imaging (MRI) or computed tomography (CT) analysis to justify treatment decisions (Pathria et al., 1987; Butler et al., 1990; Coste et al., 1994; Weishaupt et al., 1999; Kettler and Wilke, 2006). Unfortunately, these facet joint grading systems often have no reliability analysis or fail to meet the current recommendations for reliability (Little et al., 2015). However, studies assessing the validity of clinical imaging based grading systems by comparative investiga-

\footnotetext{
* Corresponding author.

E-mail address: stefan.schwan@imws.fraunhofer.de (S. Schwan).
} 
tions with microstructural data have been completed for other joints, though rarely for lumbar facet joints (Berteau et al., 2015; Gersing et al., 2016; Hawellek et al., 2016; Reina et al., 2016; Han et al., 2017).

Micro-computed tomography ( $\mu \mathrm{CT}$ ) (Cao et al., 2016) and scanning electron microscopy (SEM) are ex vivo characterization techniques that are useful in observing facet joint morphology and degeneration at a microscopic scale (Friedmann et al., 2017). The purpose of this study is to demonstrate the effective use of ex vivo microscopy methods for comparison with clinical radiographic data (MRI and CT) to provide the framework for future improved validation of lumbar facet joint grading systems that evaluate accuracy of diagnosis rather than inter- and intraoperative agreement alone. Morphological changes in the facet joint due to degeneration are analyzed using $\mu \mathrm{CT}$ and SEM to provide greater knowledge of the pathology of degradation and its impacts at the micro-scale.

\section{Materials and methods}

\subsection{Patients and samples}

All tissue samples were collected as part of a clinical study, which was approved by the Federal Ethics Committee of the medical faculty at Martin Luther University Halle (\#2011-47).

Lumbar facet joints from 38 patients ( 25 female, 13 male) were microstructurally examined using SEM and $\mu$ CT following procedural excision during lumbar interbody fusion. Patient age ranged from 43 to 81 years old, with $79 \%$ of patients being 60 or older at the time of surgery. In addition to patient sex and age, BMI values were also documented. The analyzed portion of the facet joints is highlighted in Fig. 1. Samples were comprised of partial fragments from the edge of the facet joint surface (Fig. 1B). Given that all patients underwent lumbar interbody fusion, degenerative changes in the joint samples were to be expected. Osteoarthritis, as characterized by subchondral sclerosis, was established on the basis of joint space reduction, osteophytes, and subchondral cysts. Degenerative microstructural characteristics of the chondral layer as well as the subchondral and spongy bone structure were also analyzed.

\subsection{Sample preparation}

Facet joint samples were prepared for electron microscopy and $\mu \mathrm{CT}$. While the surgery was ongoing, samples were rinsed with phosphatebuffered saline (PBS) by an assistant directly after excision and then fixed with $2.5 \%$ glutaraldehyde in PBS. The samples were then photographed for documentation and subsequent location of the cartilage surface. Following fixation, joint samples were rinsed again in PBS and then stained with $1 \%$ osmium tetroxide dissolved in PBS. In addition to staining, osmium tetroxide has the secondary effect of fixing unsaturated lipid bonds (Hayat, 2000). Immersion in the osmium tetroxide solution lasted between 24 and $72 \mathrm{~h}$, depending on the size of each individual sample. Residual osmium was then removed via stepwise dehydration in acetone (volume percent of anhydrous acetone: $10 \%$, 30\%, 50\%, 70\%, 90\%, 100\%; $6 \mathrm{~h}$ minimum per step). Samples were then embedded in "Spurr" resin (Spurr, 1969) according to the manufacturer protocol; samples were infiltrated in volume ratio $1: 2$, $1: 1,2: 1$, and 1:0 (resin:acetone) for $24 \mathrm{~h}$ and then cured for $12 \mathrm{~h}$ at $40{ }^{\circ} \mathrm{C}$ followed by $48 \mathrm{~h}$ at $70{ }^{\circ} \mathrm{C}$. The embedded samples were ground and polished in the transverse or longitudinal section to expose the bone-cartilage cross section. The cross-sectional surface was sputter coated with a 2-3 nm layer of platinum to provide conductivity for SEM investigations and all other surfaces covered with vapor-coated graphite.

\subsection{Clinical grading systems}

\subsubsection{Pathria scale for facet joint assessment (CT based)}

The classification method presented by Pathria et al. (1987) describes the condition of the facet joints based on axial CT scans via four grades: (0) normal: healthy facet joint space; (I) mild degeneration: narrowed facet joint space, small osteophytes, mild hypertrophy of articular process; (II) moderate degeneration: narrowed facet joint space, moderate osteophytes, moderate hypertrophy of articular process, mild subarticular bone erosions; (III) severe degeneration: narrowed facet joint space, large osteophytes, severe hypertrophy of articular process, severe subarticular bone erosions, subchondral cysts.

\subsubsection{Pfirrmann grading system for disc degeneration (MRI based)}

The Pfirrmann (Pfirrmann et al., 2001) grading system is based on MRI T2 spin-echo weighted images and describes degenerative changes of the intervertebral disc by analyzing water content and intradiscal distribution patterns as well as disc height. Degeneration is described in five grades: (I) homogeneous, hyperintense white signal, normal disc height; (II) inhomogeneous, hyperintense white signal, clearly differentiable nucleus and annulus, normal disc height; (III) inhomogeneous, intermittent gray signal, unclear distinction between nucleus and annulus, normal or slightly decreased disc height; (IV) inhomogeneous, hypointense dark gray signal, no distinction between nucleus and annulus, moderately decreased disc height; (V) inhomogeneous, hypointense black signal, no difference between the nucleus and annulus, collapsed disc space.

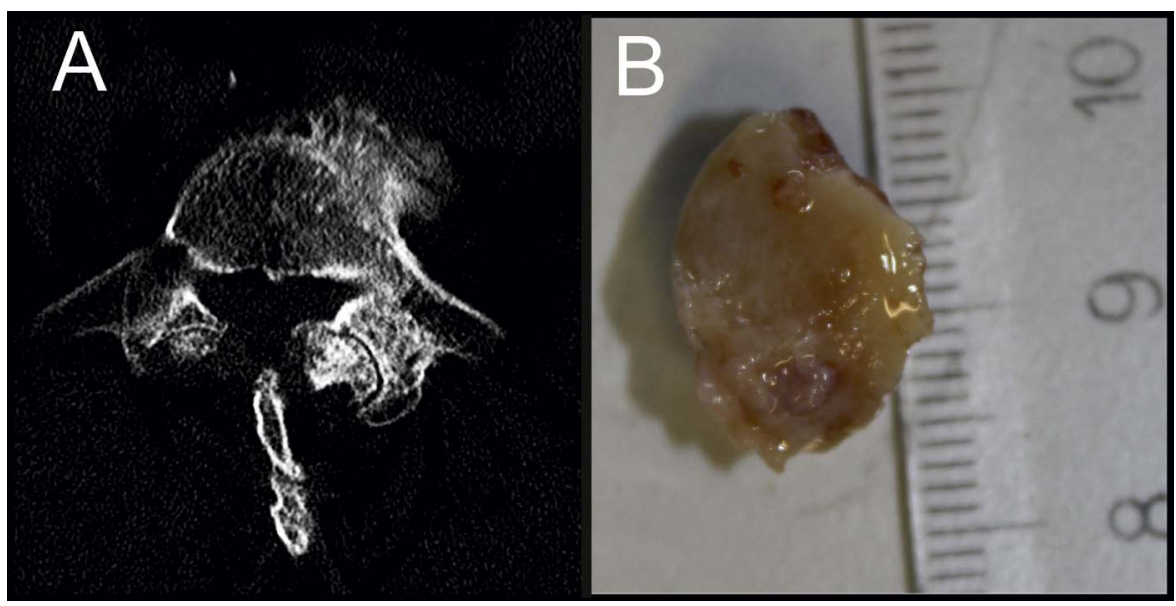

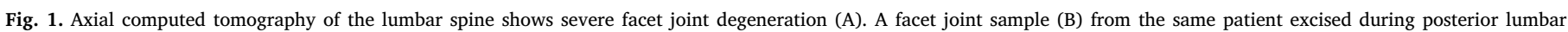
intervertebral fusion. 


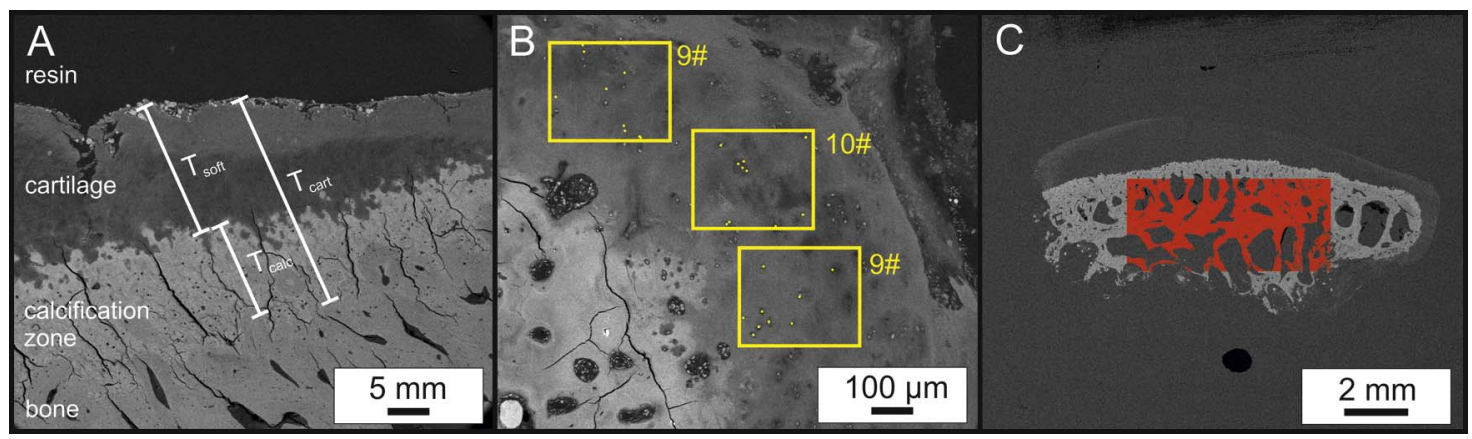

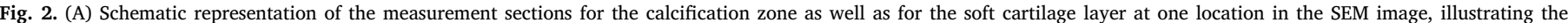

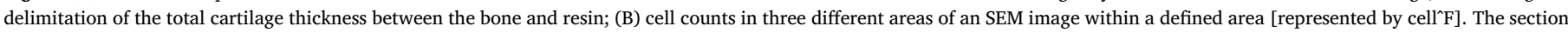

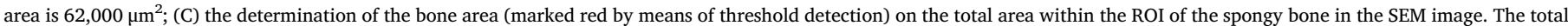

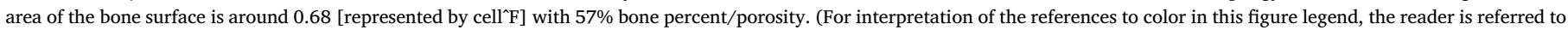
the web version of this article.)

\subsubsection{Martinek grading system for articular cartilage degeneration (histology based)}

Martinek (2003) describes articular cartilage degeneration based on histological and structural analyses. The following three grades are described: (I) matrix disruption, superficial fibrillation, increasing water content, decreased molecule size, loss of cross connections, reduced cartilage strength; (II) increased chondrocyte activity, increased metabolic activity, cell cluster formation, increased protease activity, matrix degeneration, successive loss of cartilage (Fig. 3B); (III) loss of cartilage tissue, cell death, subchondral sclerosis, subchondral cyst formation (Fig. 3C).

\subsubsection{Meyerding grading system (radiography based)}

The radiography-based Meyerding (Meyerding, 1932) spondylolisthesis classification system categorizes degeneration by the ratio of the overhanging part of the superior vertebral body in relation to anteroposterior length of the adjacent inferior vertebral body. This dislocation is described in five grades: (1) $1-25 \%$; (2) $25-50 \%$; (3) 50-75\%; (4) 75-100\%; (5) > 100\% (spondyloptosis).

\subsection{Microstructural investigations}

\subsubsection{Examination with scanning electron microscopy (SEM)}

Microstructural investigation was performed using an FEI Quanta 3D FEG (field emission gun) SEM. The backscatter detector (BSE) application was used to generate high-contrast images and minimize image artifacts. All recordings were made with a focused electron beam at an acceleration voltage of $10 \mathrm{keV}$, beam current of $43 \mathrm{pA}$, and work distance of approximately $5 \mathrm{~mm}$. Signal intensity is dependent on the chemical composition of the block sample, resulting in a material contrast image which reflects the distribution of various elements in the object. Microstructural investigation of the facet joint surfaces began be using the navigation montage mode to collate an overview image of the entire sample surface to locate areas of interest. BSE-SEM provided detailed imaging of the cellular structure and the separation of the different tissue areas of the facet joints in cross section. The various layers in the facet joint could be differentiated (cartilage, calcification zone, subchondral bone), allowing for determination of comparative layer thickness. To that end, image analysis was performed using the life science microscopy image processing software cell^₹ (Olympus Soft Imaging Solutions $\mathrm{GmbH}$ ).

\subsubsection{Elemental composition with energy dispersive X-ray spectroscopy}

Energy dispersive X-ray spectroscopy was performed on three facet joint samples using the EDX system (EDAX Inc., Mahwah, NJ, USA) integrated into the scanning electron microscope, a Jeol JSM $7401 \mathrm{~F}$ high-resolution SEM (JEOL USA, Inc., Peabody, MA, USA). This enabled qualitative investigation of the elemental composition at the bone-- cartilage interface. The samples were examined by spot analyses and surface area analyses at an acceleration voltage of $30 \mathrm{keV}$. Signal offset was determined by analysis of pure embedding resin. The osmium and phosphorus peaks were separated by unfolding the spectra in the analysis software TEAM ${ }^{\mathrm{TM}}$ EDS (EDAX Inc., Mahwah, NJ, US).

\subsubsection{Investigations with micro-computed tomography $(\mu C T)$}

The $\mu \mathrm{CT}$ unit used in this study was a phoenix nanome $\mid x 180 N F$ (GE, USA). Volume reconstruction of the $\mu \mathrm{CT}$ scans was calculated via the software program VGStudio MAX 2.2 (Volume Graphics GmbH, Heidelberg, Germany). For examining the facet joint sample blocks, image contrast was optimized by proper setting of the $\mu \mathrm{CT}$ parameters: acceleration voltage $(100 \mathrm{keV})$, radiation source current $(100 \mu \mathrm{A})$, detector sensitivity, and exposure time $(400 \mathrm{~ms})$. Eleven images were collated by the detector for each of the 500 -image pattern steps over a $360^{\circ}$ rotation. With a resulting voxel size of $25 \mu \mathrm{m}$, visualization of all tissue layers was possible with high X-ray density. Scans of biological samples with a low absorption can be improved by increasing the current in the X-ray tube.

Biological samples generally exhibit a weak absorption capacity, however trabecular bone is considered a biological hard tissue due to its calcification and is clearly visible in $\mu \mathrm{CT}$ images. Detection of soft tissues such as cartilage is possible via osmium staining. For the purposes of this study, $\mu \mathrm{CT}$ investigations served as a secondary method to validate SEM results. A partial or complete loss of cartilage on the sample surface was verified by the $3 \mathrm{D}$ image reconstruction of the joint.

\subsubsection{Thickness of the cartilage layer and calcification zone}

The cartilage layer thickness $\left(T_{\text {cart }}\right)$ and relative thickness of the calcification zone $\left(t_{\text {calc }}\right)$ of both partial and whole facet joint samples were analyzed based on the cross-sectional SEM imaging. Both parameters were measured in 20 facet joint specimens with a uniform layer of cartilage. A mean value and standard deviation was calculated for each sample based on measurements from 10 different locations on the sample's SEM image. The calcification zone was defined and measured as the interface layer between the bone and soft cartilage (Fig. 2A). The thickness of the entire cartilage layer was calculated for each sample as the sum of the mean thickness of soft cartilage and the mean thickness of the calcification zone. The relative thickness of the calcification zone was calculated as the percentage of the overall cartilage thickness. All formulas are summarized in Table 1.

\subsubsection{Cell density of the cartilage layer $\left(D_{c}\right)$}

The cell density of the soft cartilage layer was calculated by manual determination of the cell number in a defined area, averaged across three locations in each sample's collated SEM image (Fig. 2B). The defined area was constant for all three cell counts, with the 2D cell density value defined as the number of cells per unit area (equation in 
Table 1

Calculation formulas for inquiries about degeneration related tissue parameters.

\begin{tabular}{ll}
\hline Formula & Description \\
\hline$t_{\text {calc }}=\frac{T_{\text {calc }}}{T_{\text {calc }}+T_{\text {soft }}} \times 100 \%$ & $T_{\text {cart }}:$ thickness of entire cartilage layer $[\mu \mathrm{m}]$ \\
$T_{\text {cart }}=T_{\text {soft }}+T_{\text {calc }}$ & $T_{\text {calc }}:$ thickness of calcification zone $[\mu \mathrm{m}]$ \\
& $T_{\text {soft }}:$ thickness of soft cartilage $[\mu \mathrm{m}]$ \\
& $t_{\text {calc }}:$ relative thickness of the calcification zone [\%] \\
$D_{\text {cell }}=\frac{N_{\text {cell }}}{A}$ & $D_{\text {cell }}:$ cell density [cells $\left./ \mathrm{mm}^{2}\right]$ \\
& $N_{\text {cell }}:$ number of cells \\
$n_{b}=100 \%-c_{b}$ & $A:$ area [mm $\left.{ }^{2}\right]$ \\
& $n_{b}:$ trabecular bone porosity [\%] \\
& $c_{b}:$ bone portion [\%] \\
\hline
\end{tabular}

Table 1). Both the cell density and layer thickness are local measurements from each sample's SEM images, under the given assumption that this sampling is representative for the sample as a whole.

\subsubsection{Bone portion $\left(c_{b}\right)$ and trabecular bone porosity $\left(n_{b}\right)$}

To determine the area fraction of the trabeculae in the cancellous bone, bony elements were marked in the region of interest (ROI) using threshold setting (Fig. 2C) and subsequently compared to the total surface area. Measurements were collected at five different ROI's in the collated SEM image and averaged. The 2D porosity of the spongy bone is directly dependent on the flat portion of the bone, calculated as the inverse of the total area of marked trabeculae (equation in Table 1).

\section{Results}

\subsection{Patients and segment degeneration}

The patient sample population was heterogeneous (Table 2), comprised of 25 women (66\%) and 13 men (34\%). At the time of surgery, $79 \%$ of patients were 60 years or older (age range 43-81 years). 17 patients were diagnosed with Meyerding Grade 1 spondylolisthesis degeneration; the remaining 21 did not suffer from any grade of spondylolisthesis. All patients demonstrated an advanced degeneration of the intervertebral discs operated on, with 16 patients (42\%) having Pfirrmann grade IV degeneration and the remaining 22 (58\%) having Pfirrmann grade V. However, samples were obtained from spine segments with a vertebral dislocation (Grade 1, according to Meyerding) just as often as from spinal levels that showed no spondylolisthesis (Grade 0). Similarly, approximately the same numbers of samples came from collapsed motion segments (Pfirrmann Grade 5) as from motion segments with only moderately decreased disc height (Pfirrmann Grade 4) (Pfirrmann et al., 2001).

\subsection{Degradation analysis by $\mu C T$ and SEM}

The samples were analyzed across multiple $\mu \mathrm{CT}$ cross-sections and classified by their pathological degeneration according to Pathria (Pathria et al., 1987). 10 samples showed slight degeneration (Grade 1, Fig. 3A), 12 others a marked micromorphological change (Grade 2, Fig. 3B), and the remaining 16 an almost complete degeneration of the cartilage surface (Grade 3, Fig. 3C). As such, the majority of the samples (73.7\%) had Grade 2 or 3 degeneration.

The $\mu$ CT scans also showed that the angular error in the transverse or longitudinal section of the cartilage, which is critical for the correct measurement of the cartilage thickness in SEM images, was acceptable in all but 2 samples. A deviation of $90^{\circ} \pm 10^{\circ}$ between subchondral bone plate and cartilage thickness is considered to be tolerable in regard to biological material, which naturally varies in shape and size.

There was no significant correlation between uniform cartilage layer and Pfirrmann-assessed intervertebral disc degeneration grade, nor between uniform cartilage layer and Meyerding-assessed vertebral sliding grade.
Both the $\mu \mathrm{CT}$ and SEM images showed tissue damage: cartilage fragmentation and loss as well as cracks and defects within the cartilaginous surface (Fig. 4A and B). This damage results in an inhomogeneous, rough surface of the joint. The successive loss of the hyaline cartilage on the articular surface reflects the arthritic changes of the facet joint indicative of Pathria Grade II degeneration. In Pathria Grade III, the subchondral bone is no longer covered by cartilage and itself constitutes the new articular surface (Fig. 4C). This near complete cartilage loss is a characteristic feature of arthritic changes. The bone, serving as the joint surface, subsequently exhibits severe mechanical wear.

The various cancellous bone structures are distinguishable: the trabeculae, subchondral bone plate, calcification zone, and cartilage layer. The calcified cartilage layer was clearly visible, forming the transition between the bone and cartilage. This appears white in the SEM images due to the high degree of calcification. With progressive degeneration of the tissue, the calcification zone steadily grows in thickness.

\subsection{Layer thickness of the cartilage and the calcified zone}

The overall cartilage layer thickness as well as the relative thickness (\%) of the included calcification zone were determined by SEM image analysis (Fig. 1A). Due to unfavorable positioning of the tissue sample in the resin, as evident from $\mu \mathrm{CT}$ imaging, 4 samples could not be satisfactorily analyzed by SEM in terms of layer thickness. Some of the samples that showed articular cartilage in the SEM image do not have a uniform cartilage layer. In other samples the cartilage is present but severely deformed (Table 3). Tissue damage was characterized in both the SEM and $\mu \mathrm{CT}$ images by torn cartilage fragments as well as cracks and defects within the cartilaginous surface (Fig. 4). The samples with only Pathria Grade 1 and 2 degeneration have an at least approximately uniform layer of hyaline cartilage on their articular surface.

In 10 of the facet joint samples (Table 3), the calcification zone is already pathologically thickened. The calcification zone's percentage of the entire cartilage layer is greater than in healthy articular cartilage, where the calcified zone constitutes approximately one sixth (Bogduk, 2012) (about 15\%) of the cartilage thickness. The remaining 10 samples have a normal calcification zone thickness, i.e. below $15 \%$ of the total cartilage layer thickness.

On average, the female samples have a thinner cartilage layer $(740 \mu \mathrm{m} \pm 140.5 \mu \mathrm{m})$ than that of the males. The mean cartilage thickness of the male facet joints is approximately twice as much $(1311 \mu \mathrm{m} \pm 146 \mu \mathrm{m})$. No correlation between cartilage thickness and patient age (at the time of surgery) was observed in either male or female patients.

Similarly, there is no significant dependence of cartilage thickness on patient BMI. There are facet joint samples of overweight patients (BMI $\geq 25)$ as well as those of normal weight (BMI $<25)$ that have very thin cartilage layers (below $1000 \mu \mathrm{m}$ ). Similarly, there are also samples in both BMI groups which have a relatively well-preserved cartilage layer. The same applies to the cartilage thickness of the samples within the overweight range for the differentiation of nonobesity $(n=8,25 \leq$ BMI $<30)$ and obesity $(n=8$, BMI $\geq 30)$. However, the samples with a strongly deformed or even absent layer of the articular cartilage are almost exclusively those of overweight patients. Of note, 13 of the 17 patients whose facet joints lack a uniform cartilage layer are female.

\subsection{Surface cell density and cellular alteration of the cartilage}

The surface cell density of the chondrocytes in the articular cartilage was determined by SEM image analysis. Cell counting was impossible for the facet joint samples without a cartilage layer or those without recognizable cartilage cells, as seen in the SEM image. As such, these sample were excluded from this particular analysis and surface cell 
Table 2

Clinical starting position of the excised samples. For weight classification applies: 0 normal weight; 1 overweight; 2 obesity

\begin{tabular}{|c|c|c|c|c|c|c|c|}
\hline Sample & Sex & Age & Disc level & BMI & Weight classification & Disc degeneration by Pfirrmann et al. (2001) & Spondylolisthesis by Meyerding (1932) \\
\hline 01 & $\mathrm{~F}$ & 43 & L4/L5 & 24.77 & 0 & 4 & 0 \\
\hline 02 & M & 46 & $\mathrm{~L} 5 / \mathrm{S} 1$ & 23.77 & 0 & 4 & 1 \\
\hline 03 & $\mathrm{~F}$ & 76 & L4/L5 & 25.64 & 1 & 4 & 1 \\
\hline 04 & $\mathrm{~F}$ & 76 & L4/L5 & 30.46 & 2 & 5 & 1 \\
\hline 05 & M & 72 & L4/L5 & 28.09 & 1 & 5 & 0 \\
\hline 06 & $\mathrm{~F}$ & 70 & L4/L5 & 24.92 & 0 & 4 & 0 \\
\hline 07 & M & 68 & L4/L5 & 30.10 & 2 & 5 & 1 \\
\hline 08 & M & 70 & L4/L5 & 31.67 & 2 & 4 & 0 \\
\hline 09 & $\mathrm{~F}$ & 74 & L4/L5 & 32.90 & 2 & 4 & 1 \\
\hline 10 & $\mathrm{~F}$ & 62 & L4/L5 & 38.97 & 2 & 4 & 0 \\
\hline 11 & $\mathrm{~F}$ & 57 & L4/L5 & 20.55 & 0 & 4 & 1 \\
\hline 12 & $\mathrm{~F}$ & 77 & L4/L5 & 28.13 & 1 & 5 & 1 \\
\hline 13 & $\mathrm{~F}$ & 74 & L4/L5 & 32.42 & 2 & 5 & 0 \\
\hline 14 & $\mathrm{~F}$ & 72 & L4/L5 & 36.98 & 2 & 4 & 1 \\
\hline 15 & $\mathrm{~F}$ & 80 & L5/S1 & 24.09 & 0 & 4 & 0 \\
\hline 16 & M & 64 & L4/L5 & 29.63 & 1 & 4 & 0 \\
\hline 17 & $\mathrm{~F}$ & 48 & L4/L5 & 26.90 & 1 & 4 & 1 \\
\hline 18 & M & 76 & L2-S1 & 30.45 & 2 & 5 & 0 \\
\hline 19 & $\mathrm{~F}$ & 72 & L2/L3 & 27.85 & 1 & 4 & 0 \\
\hline 20 & M & 80 & L3/L4 & 29.41 & 2 & 4 & 1 \\
\hline 21 & $\mathrm{~F}$ & 76 & L3/L4 & 25.21 & 1 & 5 & 0 \\
\hline 22 & $\mathrm{~F}$ & 70 & L4/L5 & 24.01 & 0 & 4 & 0 \\
\hline 23 & $\mathrm{~F}$ & 81 & L3/L4 & 28.05 & 1 & 5 & 0 \\
\hline 24 & $\mathrm{~F}$ & 58 & L5/S1 & 31.60 & 2 & 5 & 0 \\
\hline 25 & $\mathrm{~F}$ & 61 & L4/L5 & 34.22 & 2 & 5 & 1 \\
\hline 26 & M & 66 & L3/L4 & 33.21 & 2 & 4 & 1 \\
\hline 27 & $\mathrm{M}$ & 60 & L4/L5 & 27.31 & 1 & 4 & 0 \\
\hline 28 & $\mathrm{~F}$ & 71 & L4/L5 & 26.17 & 1 & 5 & 1 \\
\hline 29 & M & 50 & L4/L5 & 31.44 & 2 & 4 & 0 \\
\hline 30 & $\mathrm{~F}$ & 75 & L4/L5 & 26.57 & 1 & 4 & 0 \\
\hline 31 & $\mathrm{~F}$ & 55 & L5/S1 & 22.49 & 0 & 5 & 0 \\
\hline 32 & $\mathrm{~F}$ & 57 & L4/L5 & 33.95 & 2 & 5 & 1 \\
\hline 33 & $\mathrm{~F}$ & 62 & L4/L5 & 28.26 & 1 & 5 & 1 \\
\hline 34 & M & 71 & L4/L5 & 24.28 & 0 & 4 & 0 \\
\hline 35 & M & 81 & L3/L4 & 24.11 & 0 & 5 & 0 \\
\hline 36 & M & 64 & L4/L5 & 26.45 & 1 & 4 & 0 \\
\hline 37 & $\mathrm{~F}$ & 75 & L3/L4 & 28.19 & 1 & 5 & 1 \\
\hline 38 & $\mathrm{~F}$ & 60 & L4/L5 & 37.33 & 2 & 4 & 1 \\
\hline
\end{tabular}

density was determined for the remaining 20 samples (Fig. 5).

All samples demonstrated the formation of cell clusters, making cell density very inhomogeneous over the sample cross-section and resulting in relatively large scattering of the measurements.

Within the cartilage layer there are local calcifications of the tissue through which cell clusters build up extracellular matrix with strong material contrast (Figs. 4-6).

\subsection{Trabecular portion and porosity of the spongy bone}

Trabecular bone portion and cancellous bone porosity were determined for each facet joint sample (Table 4).

The bone portion varies between $19.57 \%$ and $70.93 \%$, with a mean value of $47.95 \% \pm 11.71 \%$. The average porosity of the samples is $52.04 \% \pm 11.71 \%$, ranging from $29.07 \%$ to $80.53 \%$. No significant correlation between either trabecular bone portion or cancellous bone porosity to patient sex, age, or BMI were found within the sample population group.

\subsection{EDX analysis}

EDX was performed on the samples in tandem with SEM imaging (Fig. 6A) in the boundary layer between bone and cartilage, i.e. the calcification zone. As expected, increased calcium and phosphorus content is present in the areas with a high SEM material contrast. The proportion of the osmium contrast medium is negligibly small in this context.

Across all investigated samples, the calcium content is highest in the region of the calcification zone, at $13.12 \%$ (Fig. 6(1)). There is hardly any calcium present in the damaged cartilage tissue region (Fig. 6(2)). Additionally, the subchondral bone region has a lower calcium content (9.53\%) than the calcification zone. The phosphorus to calcium ratio of 1.42 suggests the presence of calcium phosphate in both areas. In samples with Pathria Grade 2 or 3 microstructural damage, the remaining cartilage layers have increased material contrast. In contrast to the cartilage tissue, the proportion of calcium and phosphorus in the direct vicinity (Fig. 6(3) and (6)) of the cells (Fig. 6(4) and (5)) is greatly increased and approximately corresponds to that of the calcification zone. For the two areas analyzed on the facet joint samples, the EDX values for calcium and phosphorus are in the same order of magnitude.

\section{Discussion}

\subsection{General aspects}

Hyaline articular cartilage in adults is devoid of any blood supply, lymphatic drainage, or innervation. Moreover, the chondrocytes are shielded by a broad extracellular matrix from both cellular repair mechanisms and the supply of nutritive substances originating from the synovial fluid. Pluripotent cells, which can be found in muscular tissue for the purpose of regeneration, are not present in articular cartilage. The reaction of cartilage tissue to injury differs from the classical threephase trauma healing associated with vascular tissue (necrosis, inflammation, and repair). Due to cartilage's avascularity, there is no migration of inflammatory cells to the site of injury. Cartilage tissue 


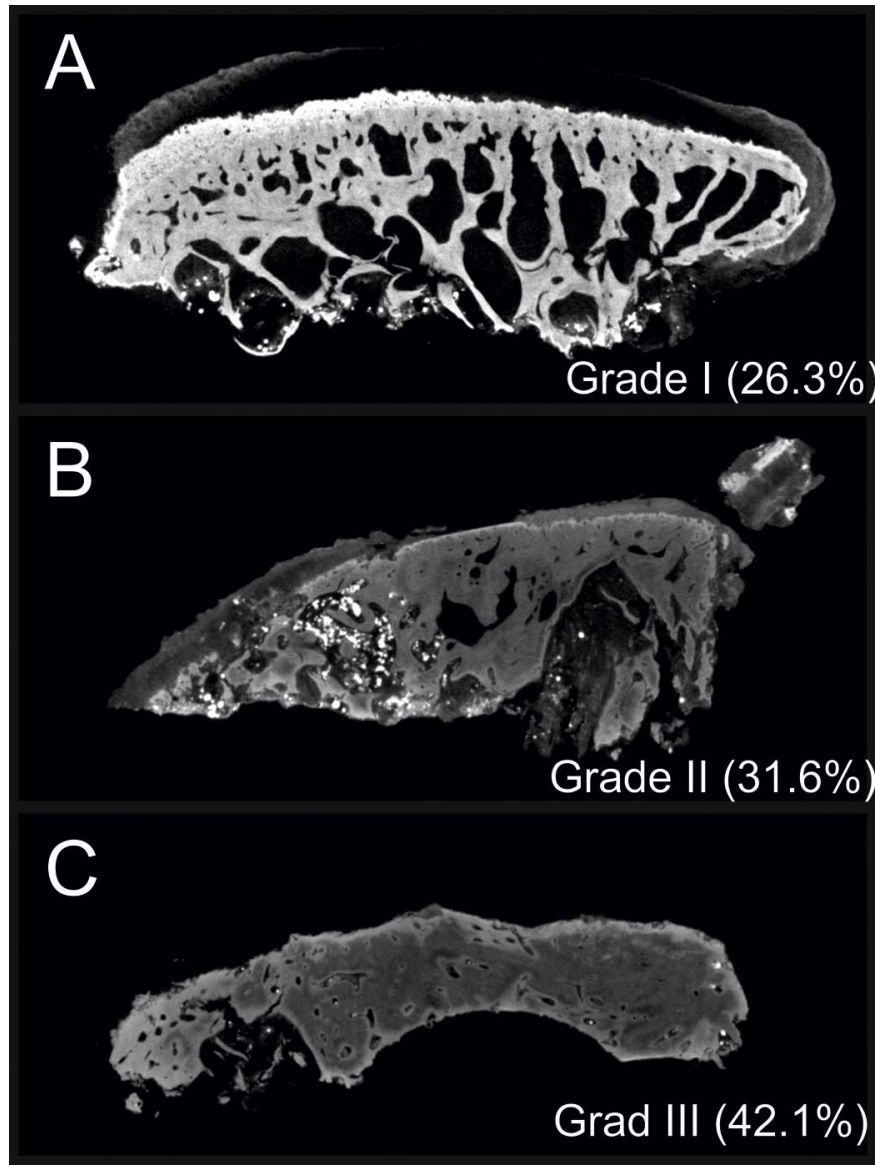

Fig. 3. $\mu \mathrm{CT}$ analysis of facet joint samples according to Pathria. (A) Almost continuously preserved cartilage layer; (B) Grade 2 with successive cartilage loss; and (C) Grade 3 with near complete cartilage loss.

contains natural inhibitors that block vessel and macrophage infiltration. Cartilage does however possess chondrocytes, but these are not able to migrate to damaged areas because they are "trapped" in a matrix of collagen and proteoglycans. To generate additional knowledge and more detailed descriptions of human facet joints, this study analyzed excised facet joint samples $(n=38)$ in regard to the calcification zone, cartilaginous cell density, and structural changes of subchondral bone. These parameters were then evaluated according the classifications of Martinek and Pathria for articular surface degeneration. CT scans were assessed to characterize structural impairments such as cracks and separation defects as well as the general loss of homogeneity. Additionally, EDX analyses verified identification of calcification zones by determining elemental calcium and phosphorus concentration.
Table 3

Layer thickness measurement results.

\begin{tabular}{|c|c|c|c|c|}
\hline Sample & $\begin{array}{l}T_{\text {cart }} \\
{[\mu \mathrm{m}]}\end{array}$ & $\begin{array}{l}T_{\text {calc }} \\
{[\mu \mathrm{m}]}\end{array}$ & $\begin{array}{l}t_{\text {calc }} \\
{[\%]}\end{array}$ & Pathological damage \\
\hline 01 & 396 & 60 & 15 & - \\
\hline 02 & 1532 & 211 & 14 & - \\
\hline 06 & 495 & 41 & 8 & - \\
\hline 07 & 1761 & 182 & 10 & - \\
\hline 08 & 1366 & 50 & 4 & - \\
\hline 11 & 924 & 449 & 49 & $\mathrm{X}$ \\
\hline 13 & 654 & 85 & 13 & - \\
\hline 15 & 1296 & 309 & 24 & $\mathrm{X}$ \\
\hline 16 & 232 & 89 & 38 & $\mathrm{X}$ \\
\hline 17 & 550 & 108 & 20 & $\mathrm{X}$ \\
\hline 18 & 1523 & 353 & 23 & $\mathrm{X}$ \\
\hline 19 & 545 & 60 & 11 & - \\
\hline 20 & 1340 & 139 & 10 & - \\
\hline 22 & 427 & 75 & 18 & $\mathrm{X}$ \\
\hline 32 & 1143 & 145 & 13 & - \\
\hline 34 & 1388 & 59 & 4 & - \\
\hline 35 & 1054 & 640 & 61 & $\mathrm{X}$ \\
\hline 36 & 1589 & 299 & 19 & $\mathrm{X}$ \\
\hline 37 & 722 & 130 & 18 & $\mathrm{X}$ \\
\hline 38 & 962 & 179 & 19 & $X$ \\
\hline
\end{tabular}

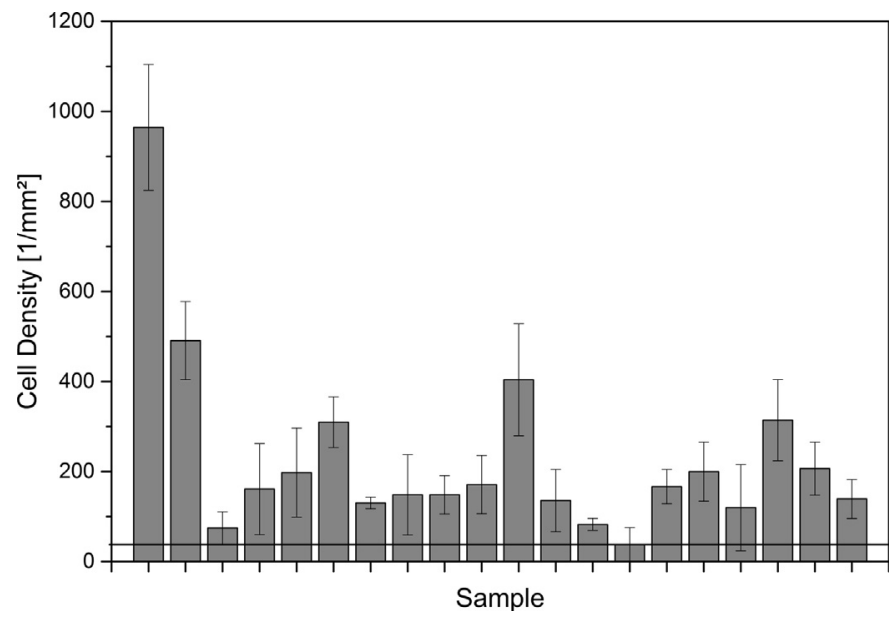

Fig. 5. Cell density for facet joint samples. The line indicates the threshold value for a pathologically increased cell number at $>45 \mathrm{~mm}^{-2}$ (Stockwell, 1967).

\subsection{Facet joint degeneration}

All samples showed microstructural degeneration of the articular surface, which supports the clinical CT assessment using the Pathria scoring system. From this score, all articular surfaces were evaluated with Grade I or worse degeneration. The examined facet joint specimens were assigned to three grades of arthritic chondral degeneration based on the cellular and morphological characteristics of the cartilage

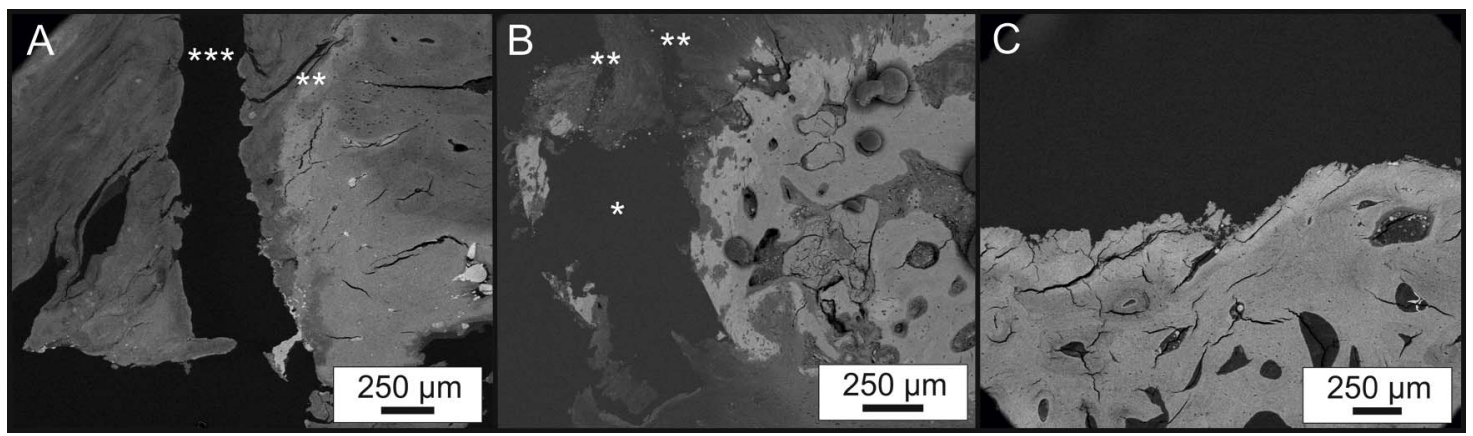

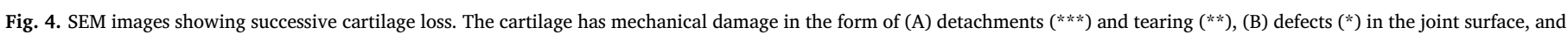
(C) complete cartilage loss with subchondral bone as the bare joint surface. 

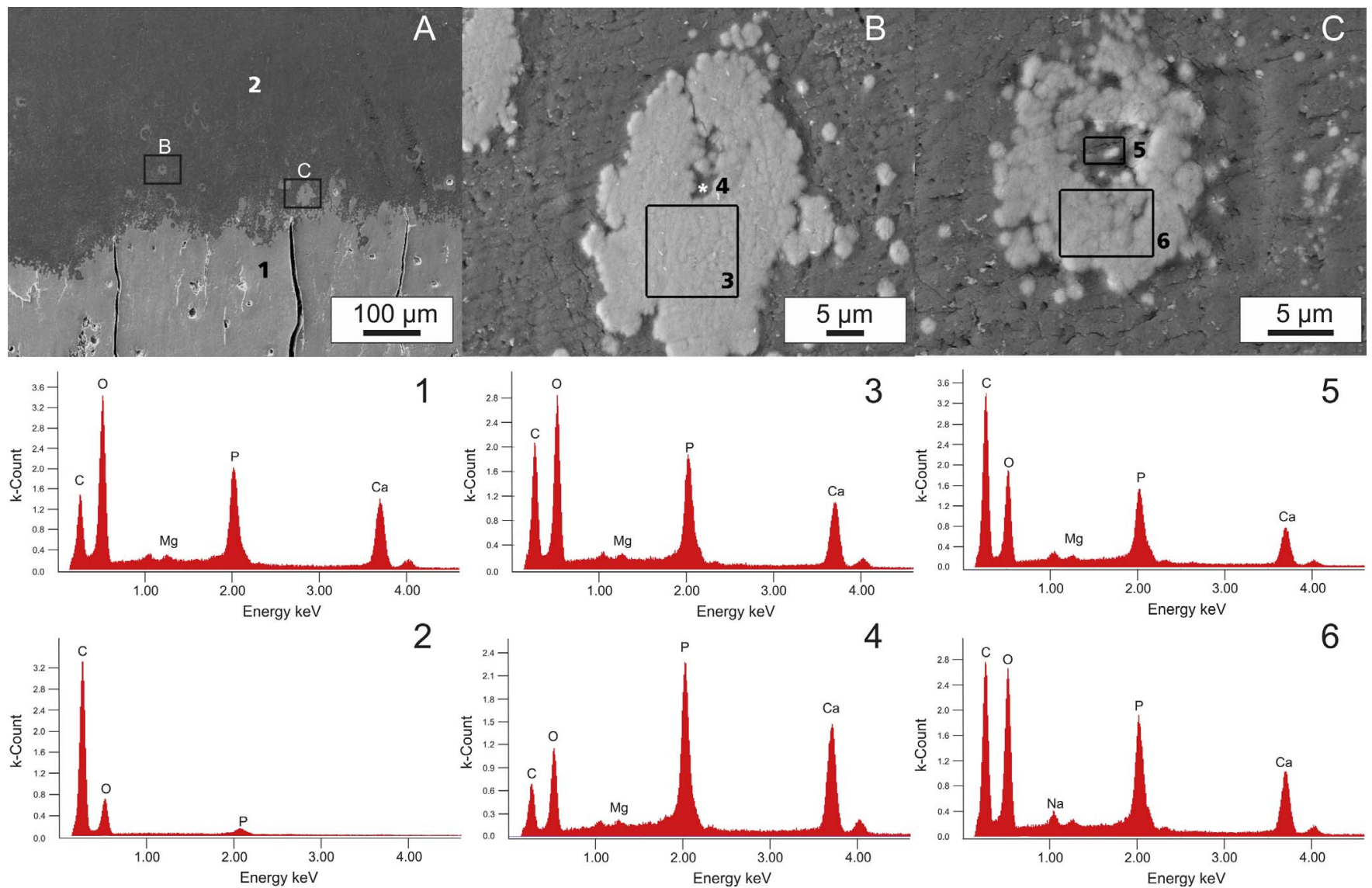

Fig. 6. The SEM images A, B, and C show the areas of EDX analysis. Calcification zone (1), cartilage tissue (2) Osteocytes (4) and (5) calcify their environment (3) and (6).

Table 4

Determination of the bone content and the porosity of the cancellous bone for all facet joint samples in area proportion.

\begin{tabular}{llllll}
\hline Sample & $\begin{array}{l}\text { Bone content } \\
{[\%]}\end{array}$ & Porosity [\%] & Sample & $\begin{array}{l}\text { Bone content } \\
{[\%]}\end{array}$ & Porosity [\%] \\
\hline 01 & 39.25 & 60.75 & 20 & 43.46 & 56.54 \\
02 & 40.19 & 59.81 & 21 & 40.17 & 59.83 \\
03 & 52.18 & 47.82 & 32 & 46.10 & 53.90 \\
04 & 61.73 & 38.27 & 23 & 36.38 & 63.62 \\
05 & 45.49 & 54.51 & 24 & 43.27 & 56.73 \\
06 & 40.91 & 59.09 & 25 & 39.60 & 60.40 \\
07 & 42.40 & 57.60 & 26 & 39.55 & 60.45 \\
08 & 60.04 & 39.96 & 27 & 37.10 & 62.90 \\
09 & 69.15 & 30.85 & 28 & 47.81 & 52.19 \\
10 & 70.93 & 29.07 & 29 & 70.92 & 29.08 \\
11 & 41.65 & 58.35 & 30 & 53.42 & 46.58 \\
12 & 53.54 & 46.46 & 31 & 60.83 & 39.17 \\
13 & 40.46 & 59.54 & 32 & 42.39 & 57.61 \\
14 & 42.00 & 58.00 & 33 & 38.35 & 61.65 \\
15 & 64.54 & 35.46 & 34 & 52.75 & 47.25 \\
16 & 67.44 & 32.56 & 35 & 36.39 & 63.61 \\
17 & 43.63 & 56.37 & 36 & 59.82 & 40.18 \\
18 & 56.96 & 43.04 & 37 & 46.23 & 53.77 \\
19 & 35.73 & 64.27 & 38 & 19.47 & 80.53 \\
& & & & &
\end{tabular}

tissue. The hyaline cartilage within a joint is composed of layers that vary, especially in regards to thickness, cell density, and matrix composition. The soft cartilage consists of the superficial, intermediate, and radial zone and is influenced by degeneration from both of its boundary surfaces. The superficial zone is damaged by mechanical impacts from torsion, extension, and compression of the spine. Abrasions can extend to the deeper layers of the intermediate zone and even into the radial zone. Based on the internal bony structures, the "tidemark," or interface between the deep zone and calcified cartilage, is ruptured and the radial zone is calcified (Mansour, 2009). These effects lead to thinning of the soft cartilage.

\subsection{Segment degeneration}

None of the specimens possessed a healthy articular surface. This was unsurprising given that all facet joint samples were excised during a spinal fusion procedure due to existent spine disease. All patients showed Pfirrmann grade IV or V intervertebral disc degeneration and decreased disc height. The reduced disc height seen in all affected motion segments increases the impact load on the dorsal column, which is supported by the facet joints. When disc degeneration and height loss become severe, there is a radical shift in compressive load bearing from the vertebral body to the neural arch. High, abnormal load bearing in these joints is closely associated with cartilage loss and osteoarthritis (Ivicsics et al., 2013). The present data support the presumption of increased facet joint degeneration following disc degeneration (Ivicsics et al., 2013). Alternatively, severe facet joint damage results in increased loading of the corresponding disc and may accelerate its degeneration. Degenerative changes in the disc are almost always accompanied by osteoarthritic alterations of the facet joints. Many biomechanical and histological studies have highlighted the close functional relationship between lumbar discs and their associated facet joints, and it is conceivable that their degenerative changes are interdependent. However, separation of cause from effect still remains controversial and is discussed at length in literature. A recent histological investigation in sheep (Moore et al., 1996) has clearly defined the possible development of facet joint arthritis as a response to disc degeneration. Butler et al. also concluded that discs degenerate before facet joints based on assessments of disc degeneration by MRI and facet joint arthritis by CT. On the other hand, other studies have not confirmed this relationship, as facet joints with marked cartilage 
damage and no detectable associated disc degeneration have been reported. The present study shows that in segments with a Pfirrmann grade IV and V, severe degeneration with a diffuse deterioration of the cartilage surface is observed. However, for further verification of a significant correlation between Pfirrmann grade and the degree of degeneration of the facet joint articular surface, a higher number of samples would be necessary.

\subsection{Surface abrasion}

The biomechanics of the evaluated joints is altered fundamentally. Healthy facet joints perform an almost frictionless sliding movement thanks to the physical characteristics of the cartilaginous surfaces and the lubricating synovial fluid. Due to the progressive loss of cartilage, a inhomogeneous and uneven surface forms. Consequently, the sliding of the articular surfaces is impeded and friction-reducing systems are disturbed. The high roughness broadens the articular surface and alters tribological qualities. This increased friction further aggravates the joint and causes worse abrasions. The cartilage layer becomes thinner and thinner until the cartilage is almost entirely removed, leaving an articular surface consists solely of the subchondral bone. Eventually, articular movement grinds directly on the surface of innervated bone. This results in every movement causing pain due to the direct application of load onto bony tissue. The progressive degeneration also leads to a dysfunction of biochemical processes in the cartilage. The samples analyzed show various degrees of cartilage degeneration. Twenty-one samples had a homogenous, though thinned cartilage layer thickness, ten samples showed a segmental loss of cartilage, and the remaining seven samples had nearly no cartilage surface left.

\subsection{Cellular density}

Healthy cartilage has a relatively low concentration of cells, representing only $5 \%$ of the total tissue volume. In degenerated cartilage however, the cellular number increases as cells proliferate and form clusters. These cells are then unable to move within the interwoven collagen fibers and proteoglycans of the extracellular matrix and remained trapped in place. The increased cell density represents the autonomic attempt to counteract arthritic changes and compensate for cartilage damage and is therefore a reliable marker of degeneration.

The facet joint belongs to the diarthroses group (Netter, 2014) and the overall structure of the zygapophyseal cartilage surface is comparable to various other human joints such as the femoral head (Mansour, 2009). As such, the cell density should also be comparable. In 1967 Stockwell determined the relevant healthy threshold at 45 cells $/ \mathrm{mm}^{2}$. Zanker et al. described the cell density of the medial condyle as a standard human articular cartilage with 45 cells $/ \mathrm{mm}^{2}$ (Zanker, 2001). The SEM image of cartilage in a progressed degenerative state shows an excess of cells and an increase cellular density. This type of uncontrolled proliferation can be found in almost all of the examined specimens. In our analyses, seven samples showed a complete loss of the cartilage surface, representing late-stage degeneration. As such, determination of cell density was not possible. The cartilage layer of only one sample showed a normal cell density, belonging to an 81-yearold person of normal weight. The other samples show an increased cell density in 19 of 20 cases, which is characteristic of arthritic articular cartilage. Although the articular process exhibits a homogeneous cartilage layer, it also already shows areas of increased calcification. In light of this, we assume that the chondrocytes have already exceeded the threshold for pathological proliferation. At this stage of degeneration, which correlates to Grade 2 according to Pathria, the facet joint is usually ossified heavily to severely limit joint movement. Due to the lack of loading on the joint, the articular surface is not engaged and there is no mechanical abrasion. As such, the cartilage layer remains nearly undamaged.

\subsection{Subchondral sclerosis}

Another criterion for osteoarthritis is subchondral sclerosis, which results in the progressive calcification of the cartilage. As the calcification zone widens, making up $15 \%$ or more of the cartilage layer, the adjacent bone condenses and the cartilage becomes much more rigid (Berteau et al., 2013). These processes are interdependent and occur simultaneously. The sole purpose of the calcification zone is to secure the articular cartilage to the bone surface. As already mentioned above, hyaline cartilage is associated with a limited ability to regenerate. Superficial injuries to the cartilaginous tissue, which typically occur far from the bone surface, show no tendency to heal and there is no observable, effective repair process. Instead the defect persists, broadens due to mechanical abrasion, and may lead to an arthrosis of the corresponding joint. The superficial zone of the cartilage erodes and disappears. The abrasion of the cartilage may also breach the deeper zones. In cases where the damage affects not only the cartilage but also the bone, a distinct repair process is initiated. Osteocytes immigrate to the cartilage and replace the defect with granulation tissue. The "tidemark" is penetrated and the radial zone becomes calcified, increasing the overall thickness of the calcified zone. The soft cartilage, which serves as a load-bearing cushion during facet joint movement, loses substance. It thins and loses the ability to effectively compensate and transmit loads, resulting in poor shock absorption of impacts. As the total cartilage volume decreases, the mechanical pressure on the remaining tissue increases proportionally. The rigidification of the tissue lessens both its elasticity and plasticity, leading to cuts and cracks in the cartilage as well as the bone tissue. The articular surface gradually deteriorates from degenerative changes of the tissue. This decrease in the cartilage layer amplifies the impact on the subchondral bone as well as the cancellous bone. Due to the modified load transmission, a restructuring of the bone occurs at the trabecular level. The bony structure is remodeled according to the warped angles in the joint and a new architecture is generated based on the shifted loads (Kawcak et al., 2001). The cancellous bone is stabilized by the generation of additional trabecular mesh in order to offer impact resistance. This leads to increased trabecular volume, which also influences the characteristics of the cancellous bone, increasing its rigidity and decreased plasticity. As a result, the cancellous bone is no longer able to absorb the applied forces effectively. The relative trabecular fraction (BV/TV) of physiologically healthy joints is between $22.5 \%$ and $35 \%$ (Zanker, 2001). For the two segments forming the facet joints, the inferior articular process has a higher bone volume than the superior articular process. The bone portion of the cancellous bone gradually decreases moving from the upper to the lower joint region (Zanker, 2001). None of the samples showed a trabecular fraction in the normal range of 22.5-35\% (Zanker, 2001); most samples had an increased BV/TV. Half of the samples $(n=18,49 \%)$ had a bone fraction of $35-45 \%$. One sample (F48), which showed a homogenous cartilage layer and a thickened calcification zone, actually had a decreased cancellous bone fraction. This sample is from a 60 -year-old female with Grade 2 adiposity who belongs to the risk group for osteoporosis. In this case, the body was not able to adapt to the modified load conditions and an increased degradation of the bone led to a less dense trabecular mesh. Due to the decreased trabecular mesh density the bone has diminished rigidity, which results in a heightened susceptibility for pathological fractures. Twenty samples showed an enlarged calcification zone between the soft cartilage and subchondral spongy bone. We assess this as Grade III degeneration according to Martinek. However, there was no significant correlation between age, gender, or BMI as is known to others joints (Wilke et al., 2012; Reina et al., 2016). The samples excluded due to loss of the calcification zone were most commonly from female (76\%), elderly ( $>60$ years, $88 \%$ ), or overweight patients (94\%). 


\subsection{Energy dispersive $X$-ray spectroscopy}

EDX analysis identified the area between the cartilage and bone, contrasted in the SEM image, as the calcification zone due to the high levels of phosphorus and calcium present there. The relative calcium and phosphorus concentrations detected indicate the presence of hydroxyapatite and calcium phosphate. Hydroxyapatite is composed of calcium, phosphorus, oxygen, and hydrogen (Lee et al., 2006; Hristova et al., 2011). The stoichiometric ratio of calcium to phosphorus is $5: 3$ for hydroxylapatite (Lee et al., 2006). The ratios for calcium to phosphorus determined from the measured atomic ratio are approximately 10:7 (7.1 \pm 0.25$)$. Therefore, it may be assumed that hydroxyapatite is incorporated into both the bone and calcification zone. The presence of calcium phosphate in the calcified areas is also assumed. With progressive degeneration of the facet joints, calcification spreads to the soft cartilage tissue. The hydroxylated calcium phosphate salt hardens the cartilage and causes rigidification of the facet joint surface. The presence of fluorine, sodium, magnesium, chlorine, and potassium indicates that the calcified zone contains other mineral compounds in addition to hydroxyapatite. The occurrence of bone minerals such as calcium chloride, calcium fluoride, and magnesium phosphate is assumed since the calcification zone also contains chlorine, fluorine, and magnesium in small amounts. The EDX analysis also confirms that cells are located in the cartilage (Fig. 6B and C), which fill their immediate environment with osseous extracellular matrix (Fig. 6(3)-(6)). It can be assumed that these are bone-forming cells, which migrate into the cartilaginous tissue. The increased proportion of calcium and phosphorus indicates the incorporation of inorganic minerals as the basis for the bony, hard substance in the cartilage. Fluorine, sodium, magnesium, chlorine, and potassium have been detected as additional salts and salt-forming elements in the vicinity of the cells. The cartilage tissue hardens as a result of bony infiltration and the soft, elastic cartilage layer thins. This reduces the proportion of soft cartilage tissue, increasing the proportional thickness of the calcification zone. Thereby sclerosis occurs, which is characteristic of osteoarthritis.

\subsection{Back pain}

Kalichmann et al. found no association between back pain and degenerative features of the lumbar spine, such as intervertebral disc narrowing, facet joint osteoarthritis, and degenerative spondylolisthesis. Another study showed that microscopic changes occur in the intervertebral discs and facet joints from an early age. Both examples demonstrate that the existence of degenerative changes in the motion segments alone is not necessarily associated with low back pain. It still remains unclear why some patients with degenerative changes suffer from low back pain while others are asymptomatic. This phenomenon and the triggering factor for low back pain require further investigation.

\subsection{Limitations}

The methods employed in this study can be used to map all characteristics of degeneration. It has been shown that $\mu \mathrm{CT}$ and SEM with EDX allow a very detailed classification of individual degenerative characteristics. As such, the sample size of 38 patients is not sufficient to allow for a significant correlation of microstructural degeneration parameters to available clinical data. That means that the identification of statistically significant measurement categories is not possible. The influence of the sample material, preparation method, and the potential and limitations of the microstructural investigations on the gained results are versatile. However, all samples are degenerated human lumbar facet joints obtained from the inferior articular process. The various dependencies shown in this study can therefore be used as the basis for a complex power analysis to determine the optimal group size.

The specimens were not obtained from autopsy but were excised during surgery. Consequently, entire joints are not available for microstructural examination. Furthermore, the thickness of the cartilage depends on the depth of the surgeon's cut. If the cut is not orthogonal to the cartilaginous layer, error is introduced when measuring the cartilage thickness from the SEM images due to the angle error $\alpha$ (Fig. 6(1)). To combat this, the angle error is calculated from the $\mu \mathrm{CT}$ images. If the angle between the cut and cartilage cross section is less than $\pm 10^{\circ}$, measurement errors are minimal $(<1.5 \%)$. Such a small margin of error lies far below the values for the standard deviation of the measured cartilage thicknesses. Therefore, the calculated angle errors are not included. The complete analysis of the images was conducted manually, therefore human error from individual assessment is always a possibility as identical features can be interpreted slightly differently by different observers.

\section{Conclusion}

Known degenerative features of the articular cartilage can also be seen at the human facet joint. In order to make a definitive statement about the dependence of the micromorphological degeneration on relevant clinical data, as well as about the microstructural parameters themselves, the number of the analyzed samples needs to be increased.

Human facet joint samples were successfully characterized with SEM, $\mu \mathrm{CT}$, and EDX. These techniques allow for high-resolution assessment of morphological features of facet joint degeneration. Nearly all samples demonstrated high-grade structural damage, including cartilage layer erosion from fragmentation and cracking, a pathologically thickened calcification zone, local calcification sites, and cell cluster formation. The results of the microstructural examinations confirmed the findings of clinical imaging, i.e. computed tomography and magnetic resonance imaging. Furthermore, the applied methodologies allow for more precise statements on the degenerative micromorphological changes of facet joints. The comparison of these techniques can lead to more accurate conclusions from clinical imaging. In order to make a significant statement about micromorphological degeneration's dependence on the clinically relevant data, as well as about the microstructural parameters among themselves, the number of the analyzed samples needs to be increased.

\section{Conflict of interest}

None of the authors have potential conflicts of interest.

\section{Acknowledgments}

The authors are grateful to W. Petzold, U. Heunemann, and N. Teuscher from Fraunhofer IMWS for technical assistance as well as to the neurosurgical staff of the BG Kliniken Bergmannstrost for cooperation. We also extend our special thanks to all patients who consented to their tissue being used for research purposes. This work was supported by the German Federal Ministry of Education and Research (BMBF, PtJBio, 0315883) and the Whitaker Biomedical Engineering Research Fellowship.

\section{References}

Berteau, J.-P., Mielke, G., Morlock, M.M., Huber, G., 2015. Morphological and biomechanical analyses of the subchondral mineralized zone in human sacral facet joints: application to improved diagnosis of osteoarthritis. Clin. Anat. 28, 538-544. Berteau, J.-P., Nover, L., Mielke, G., Ivicsics, M., Morlock, M.M., Huber, G., 2013. In vitro characterisation of the biomechanical properties of the subchondral mineralised zone of lumbosacral facet joints. Comput. Methods Biomech. Biomed. Eng. 16, 226-227. Bogduk, N., 2012. Clinical Anatomy of the Lumbar Spine and Sacrum. Elsevier Health Sciences.

Butler, D., Trafimow, J.H., Andersson, G.B.J., Mcnell, T.W., Huckman, M.S., 1990. Disks degenerate before facets. Spine 15, 111-113.

Cao, Y., Zhang, Y., Yin, X., Lu, H., Hu, J., Duan, C., 2016. 3D visualization of the lumbar facet joint after degeneration using propagation phase contrast micro-tomography. 
Sci. Rep. 6.

Coste, J., Judet, O., Barre, O., Siaud, J.R., Delara, A.C., Paolaggi, J.B., 1994. Interobserver and intraobserver variability in the interpretation of computed-tomography of the lumbar spine. J. Clin. Epidemiol. 47, 375-381.

DiPaola, C.P., Molinari, R.W., 2008. Posterior lumbar interbody fusion. J. Am. Acad. Orthop. Surg. 16, 130-139.

Friedmann, A., Goehre, F., Ludtka, C., Mendel, T., Meisel, H.-J., Heilmann, A., Schwan, S., 2017. Microstructure analysis method for evaluating degenerated intervertebral disc tissue. Micron 92, 51-62.

Gellhorn, A.C., Katz, J.N., Suri, P., 2013. Osteoarthritis of the spine: the facet joints. Nat. Rev. Rheumatol. 9, 216-224.

Gersing, A.S., Schwaiger, B.J., Heilmeier, U., Joseph, G.B., Facchetti, L., Kretzschmar, M., Lynch, J.A., McCulloch, C.E., Nevitt, M.C., Steinbach, L.S., Link, T.M., 2016. Evaluation of chondrocalcinosis and associated knee joint degeneration using MR imaging: data from the osteoarthritis initiative. Eur. Radiol. 1-10.

Gill, K., Blumenthal, S.L., 1993. Posterior lumbar interbody fusion - a 2-year follow-up of 238 patients. Acta Orthop. Scand. 64, 108-110.

Han, B.K., Kim, W., Niu, J., Basnyat, S., Barshay, V., Gaughan, J.P., Williams, C., Kolasinski, S.L., Felson, D.T., 2017. Chondrocalcinosis in knee joints is associated with pain but not with synovitis: data from the osteoarthritis initiative. Arthritis Care Res.

Hawellek, T., Hubert, J., Hischke, S., Krause, M., Bertrand, J., Pap, T., Püschel, K., Rüther, W., Niemeier, A., 2016. Articular cartilage calcification of the hip and knee is highly prevalent, independent of age but associated with histological osteoarthritis: evidence for a systemic disorder. Osteoarthr. Cartil. 24, 2092-2099.

Hayat, M.A., 2000. Principles and Techniques of Electron Microscopy Biological Applications. Cambridge University Press.

Hoy, D., March, L., Brooks, P., Blyth, F., Woolf, A., Bain, C., Williams, G., Smith, E., Vos, T., Barendregt, J., Murray, C., Burstein, R., Buchbinder, R., 2014. The global burden of low back pain: estimates from the Global Burden of Disease 2010 study. Ann. Rheum. Dis. 73, 968-974.

Hristova, G.I., Jarzem, P., Ouellet, J.A., Roughley, P.J., Epure, L.M., Antoniou, J., Mwale, F., 2011. Calcification in human intervertebral disc degeneration and scoliosis. J. Orthop. Res. 29, 1888-1895.

Ivicsics, M.F., Bishop, N.E., Sellenschloh, K., Berteau, J.-P., Morlock, M.M., Huber, G., 2013. The effect of nucleotomy on facet joint loading - a porcine in vitro study. Clin. Biomech. 28, 853-859.

Kalichman, L., Hunter, D.J., 2007. Lumbar facet joint osteoarthritis: a review. Semin. Arthritis Rheum. 37, 69-80.

Kawcak, C.E., McIlwraith, C.W., Norrdin, R.W., Park, R.D., James, S.P., 2001. The role of subchondral bone in joint disease: a review. Equine Vet. J. 33, 120-126.

Kettler, A., Wilke, H.J., 2006. Review of existing grading systems for cervical or lumbar disc and facet joint degeneration. Eur. Spine J. 15, 705-718.
Lee, R.S., Kayser, M.V., Ali, S.Y., 2006. Calcium phosphate microcrystal deposition in the human intervertebral disc. J. Anat. 208, 13-19.

Lin, P.M., Cautllli, R.A., Joyce, M.F., 1983. Posterior lumbar interbody fusion. Clin. Orthop. Relat. Res. 154-168.

Little, J.W., Grieve, T.J., Cramer, G.D., Rich, J.A., Laptook, E.E., Stiefel, J.P.D., Linaker, K., 2015. Grading osteoarthritic changes of the zygapophyseal joints from radiographs: a reliability study. J. Manip. Physiol. Ther. 38, 344-351.

Mansour, J.M., 2009. Kinesiology The Mechanics and Pathomechanics of Human Movement. In: Oatis, C.A. (Ed.), Williams \& Wilkens, pp. 66-80.

Martinek, V., 2003. Anatomy and pathophysiology of articular cartilage. Deutsche Z. Sportmed. 54, 166-170.

Meyerding, H.W., 1932. Spondylolisthesis. J. Bone Jt. Surg. 1.

Miyakoshi, N., Abe, E., Shimada, Y., Okuyama, K., Suzuki, T., Sato, K., 2000. Outcome of one-level posterior lumbar interbody fusion for spondylolisthesis and postoperative intervertebral disc degeneration adjacent to the fusion. Spine 25, 1837-1842.

Moore, R.J., VernonRoberts, B., Osti, O.L., Fraser, R.D., 1996. Remodeling of vertebral bone after outer anular injury in sheep. Spine 21, 936-940.

Netter, F.H., 2014. Atlas of Human Anatomy, Professional Edition. Elsevier.

Okuyama, K., Abe, E., Suzuki, T., Tamura, Y., Chiba, M., Sato, K., 1999. Posterior lumbar interbody fusion - a retrospective study of complications after facet joint excision and pedicle screw fixation in 148 cases. Acta Orthop. Scand. 70, 329-334.

Pathria, M., Sartoris, D.J., Resnick, D., 1987. Osteoarthritis of the facet joints - accuracy of oblique radiographic assessment. Radiology 164, 227-230.

Pfirrmann, C.W.A., Metzdorf, A., Zanetti, M., Hodler, J., Boos, N., 2001. Magnetic resonance classification of lumbar intervertebral disc degeneration. Spine 26, 1873-1878.

Prolo, D.J., Oklund, S.A., Butcher, M., 1986. Toward uniformity in evaluating results of lumbar spine operations - a paradigm applied to posterior lumbar interbody fusions. Spine 11, 601-606.

Reina, N., Cavaignac, E., Pailhé, R., Pailliser, A., Bonnevialle, N., Swider, P., Laffosse, J.M., 2016. BMI-related microstructural changes in the tibial subchondral trabecular bone of patients with knee osteoarthritis. J. Orthop. Res.

Spurr, A.R., 1969. A low-viscosity epoxy resin embedding medium for electron microscopy. J. Ultrastruct. Res. 26, 31-43.

Stockwell, R.A., 1967. The cell density of human articular and costal cartilage. J. Anat. 101, 753-763.

Weishaupt, D., Zanetti, M., Boos, N., Hodler, J., 1999. MR imaging and CT in osteoarthritis of the lumbar facet joints. Skelet. Radiol. 28, 215-219.

Wilke, H.-J., Zanker, D., Wolfram, U., 2012. Internal morphology of human facet joints: comparing cervical and lumbar spine with regard to age, gender and the vertebral core. J. Anat. 220, 233-241.

Zanker, D., 2001. Computertomographische Untersuchungen zur Spongiosastruktur der Facettengelenke an Hals- und Lendenwirbelsäule. 